\title{
Certolizumab para el tratamiento de un paciente con artritis reumatoidea refractaria a múltiples agentes biológicos
}

\author{
Margarita Landi, Gustavo Citera \\ Instituto de Rehabilitación Psicofísica, Buenos Aires, Argentina.
}

\section{Resumen}

Hoy en día contamos con un amplio abanico de tratamientos posibles para la artritis reumatoidea, empezando por el metotrexato como droga de primera línea, además de diversas drogas modificadores de la artritis reumatoidea (DMAR) y agentes biológicos. Sin embargo, una problemática se plantea en aquellos pacientes que son no respondedores a los diversos agentes, en quienes las guías de tratamiento internacionales ya no plantean opciones. Se presenta el caso clínico de una paciente con AR con falla a múltiples DMAR y agentes biológicos de diferentes mecanismos de acción y que finalmente responde a un nuevo inhibidor de los anti-TNF (Certolizumab pegol). Se discuten las posibilidades terapéuticas en estos pacientes y los datos relacionados con el Certolizumab pegol.

\section{Abstract}

Today we have a wide range of possible treatments for rheumatoid arthritis, starting with methotrexate as first-line drug, followed by various disease modifiers rheumatoid arthritis drugs (DMARDs) and biological agents. However, a problem arises in patients who are not responding to the various drugs, where international guidelines no longer pose treatment options. We present here the case of a patient with RA that failed to multiple traditional DMARDs and biological agents of different mechanisms of action and finally responded to a new inhibitor of anti-TNF (Certolizumab-pegol). Therapeutic options for these kinds of patients and data related to Cerlolizumab-pegol are discussed.

\section{Introducción}

La artritis reumatoidea (AR) es una enfermedad inflamatoria autoinmune. Afecta al $1 \%$ de la población aproximadamente, con un marcado predominio por el sexo femenino. El concepto de ventana de oportunidad en el tratamiento de la AR es ampliamente conocido. $\mathrm{Ha}$ sido demostrado que el diagnóstico precoz y el tratamiento temprano impacta positivamente en el pronóstico y evolución de estos pacientes. En el 2013, el Colegio Americano de Reumatología (ACR) y la Liga Europea contra las Enfermedades Reumáticas (EULAR) publicaron las actualizaciones en el tratamiento de dicha patología. Hoy en día contamos con un amplio abanico de tratamientos posibles para esta enfermedad, empezando por el metotrexato como droga de primera línea, además de diversas drogas modificadoras de la artritis reumatoidea (DMAR) y agentes biológicos. Sin embargo, una problemática se plantea en aquellos pacientes que son no respondedores a los diversos agentes, en quienes las guías de tratamiento internacionales ya no plantean opciones.

\section{Caso clínico}

Paciente de sexo masculino de 58 años de edad, con antecedentes de tabaquismo de 30 paquetes/año. En octubre de 2007 es diagnosticado con artritis reumatoidea (según criterios ACR 1987 y 2010 ), de características seropositiva tanto para factor reumatoideo como para el anticuerpo péptido citrulinado cíclico (anti-CCP), erosiva, nodular, poliarticular, con compromiso de pequeñas y grandes articulaciones, de curso progresivo y sostenido. Inicia tratamiento con metotrexato en dosis crecientes hasta 30 $\mathrm{mg} / \mathrm{semana}$ y esteroides, agregando luego leflunomida. Sin embargo, por continuar con alta actividad de la enfermedad, en junio de 2008 inicia tratamiento con etanercept. En noviembre de 2010 por falla secundaria al etanercept es rotado a abatacept intravenoso a dosis usuales. Presenta buena respuesta inicial a dicha terapia, logrando suspender esteroides y leflunomida. No obstante, en noviembre de 2011 se rota nuevamente el tratamiento biológico por tocilizumab por alta actividad de la enfermedad. Dicha medicación es supendida por falla primaria y en septiembre de 2012 recibe dos pulsos de rituximab de un gramo

\section{Correspondencia}

E-mail: margarita_landi@hotmail.com 


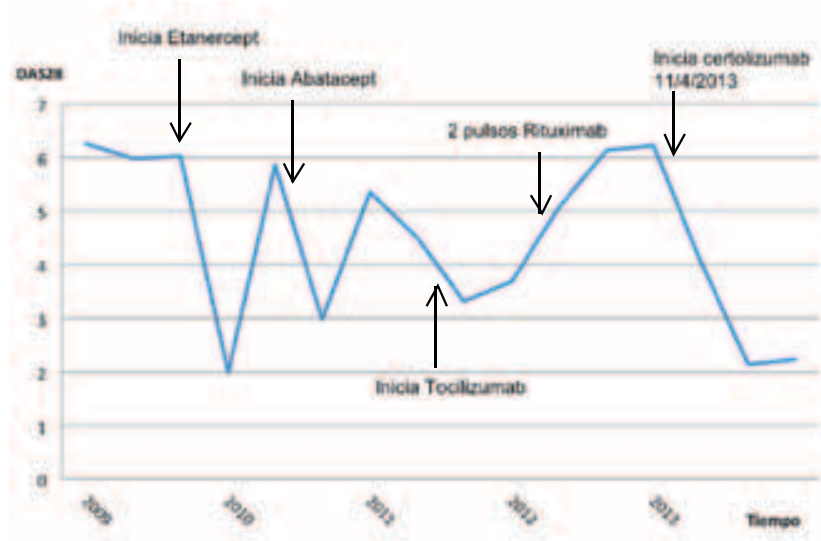

Figura 1. DAS28 a lo largo del tiempo.

cada uno sin respuesta clínica. Con una dosis acumulada de esteroides equivalente a $12,350 \mathrm{mg}$ de prednisona y por falta de respuesta a los diversos agentes biológicos, se decide iniciar tratamiento con Certolizumab $400 \mathrm{mg}$ en los días $0,15,30$ y luego $200 \mathrm{mg}$ cada 15 días en junio de 2013. El paciente logra muy buena respuesta clínica por DAS28, respuesta ACR 20, 50 y 70 logrando disminuir la dosis de prednisona de $10 \mathrm{mg} /$ día a $2,5 \mathrm{mg} /$ día. Actualmente el paciente se encuentra en clase funcional 1, no presenta articulaciones inflamadas ni dolorosas, DAS28 2.13 y HAQ-A 0.

\section{Discusión}

En la actualidad existe una problemática en pacientes con artritis reumatoidea y respuesta inadecuada a diversos agentes biológicos. A su vez, existe poca evidencia para utilizar un inhibidor del TNF alfa en pacientes tratados previamente con un biológico con otro mecanismo de acción. De la misma forma, contamos con escasa evidencia para utilizar un segundo anti-TNF luego de la falla a uno previamente, siendo los datos contradictorios. Federico Navarro-Sarabia demostró en 2009 que menos del 50\% de los pacientes presentaban buena respuesta a un segundo inhibidor del $\mathrm{TNF}^{2}$. Sin embargo, un metaanálisis en 2011 demostró que la utilización de un segundo anti-TNF puede ser clínicamente relevante ${ }^{3}$.

Certolizumab pegol es el primer anticuerpo monoclonal Fab pegilado inhibidor del TNF alfa. La unión de una cadena PEG a la porción Fab aumenta su vida media a 14 días en promedio. La falta de una porción $F c$ puede evitar posibles efectos mediados por esta porción, tales como aquellos mediados por complemento o citotoxicidad mediada por células dependiente de anticuerpos como se observa in vitro ${ }^{4}$.
Certolizumab pegol ha demostrado ser útil en el tratamiento de pacientes con AR. Disminuye la actividad de la enfermedad, mejorando la calidad de vida ${ }^{5}$, la capacidad funcional ${ }^{6,7}$, y la fatiga ${ }^{8}$, con un perfil de seguridad aceptable. Es efectivo para pacientes con AR y respuesta inadecuada a metotrexato ${ }^{9,10}$, como así también en pacientes con respuesta inadecuada a DMAR u otros agentes biológicos ${ }^{11}$. Es efectivo en forma de monoterapia ${ }^{12}$ o combinado con metotrexato. Asimismo, el tratamiento con certolizumab pegol ha logrado disminuir la progresión radiológica en pacientes con AR. En la fase de extensión del estudio RAPID1 se observó que los efectos beneficiosos de certolizumab asociado a metotrexato se sostienen en el tiempo ${ }^{13}$.

Si bien los inhibidores del TNF alfa han demostrado ser eficaces en el tratamiento de la AR, en particular cuando son asociados a metotrexato, las poblaciones en estos estudios son muy seleccionadas pudiendo no reflejar al paciente de la práctica clínica diaria ${ }^{14,15}$. En el estudio REALISTIC, la adición de certolizumab a la terapia actual de pacientes con AR activa refractaria a diversos tratamientos (incluyendo agentes biológicos de distintos mecanismos de acción y DMAR no biológicos) se asoció con una rápida respuesta clínica. El 37,6\% de los pacientes incluidos en este estudio habían recibido inhibidores del TNF alfa (10,1\% más de uno), y el 5,4\% había recibido agentes biológicos con otro mecanismo de acción. Luego de 12 semanas, el tratamiento con certolizumab se asoció con una rápida y consistente mejoría clínica, reduciendo la actividad de la enfermedad y capacidad funcional en pacientes con o sin tratamiento previo con inhibidores del TNF alfa. Si bien la eficacia de certolizumab había sido previamente demostrada en pacientes sin tratamiento previo con anti-TNF, este estudio extiende su eficacia a pacientes con tratamiento previo con inhibidores del TNF alfa, independientemente del número y tipo de inhibidor utilizado ${ }^{11}$.

Este caso clínico plantea 2 problemáticas de la consulta diaria. En primer lugar, hay escasos estudios clínicos en pacientes con AR que avalen el uso de un anti-TNF cuando ha sido utilizado previamente un agente biológico con otro mecanismo de acción. En segundo lugar, resalta la falta de datos disponibles en la terapéutica de pacientes con falla a 2 o más agentes biológicos. Si bien la actualización de las guías de tratamiento de la ACR, EULAR y SAR para la AR ha sido de gran utilidad, un problema se plantea en pacientes con falla a diversos agentes biológicos de diferente mecanismo de acción.

A modo de síntesis, en el paciente expuesto, certolizumab demostró disminuir la actividad de la enfermedad, 
mejorar la calidad de vida y capacidad funcional, disminuyendo la dosis de esteroides. Certolizumab podría ser considerado en pacientes con falla a inhibidores del TNF alfa, y quizás en pacientes con falla a más de un agente biológico de distinto mecanismo de acción. Sería interesante, evaluar su efectividad en un grupo más grande de pacientes con AR activa, refractaria a distintos agentes biológicos.

\section{Bibliografía}

1. Arnett F, Edworthy S, Bloch D, McShane D, Fries J, Cooper N, et al. The American Rheumatism Association 1987 revised criteria for the classification of rheumatoid arthritis. Arthritis Rheum 1988; 31:315-24.

2. Federico Navarro-Sarabia, Dolores Ruiz-Montesinos, Blanca Hernandez, Victoria Navarro-Compán, Sara Marsal, Mireia Barcelo, Eva Perez-Pampín and Juan J Gómez-Reino. DAS-28-based EULAR response and HAQ improvement in rheumatoid arthritis patients switching between TNF antagonists. BMC Musculoskeletal Disorders 2009, 10:91 doi:10.1186/1471-2474-10-91.

3. Remy A, Avouac J, Combe B. Clinical relevance of switching to a second tumor necrosis factor-alpha inhibitor after discontinuation of a first tumor necrosis factor-alpha inhibitor in rheumatoid arthritis: A systematic literature review and meta-analysis. Clin Exp Rheumatol 2011; 29:96-103.

4. Nesbitt A, Fossati G, Bergin M, Stephens P, Stephens $S$, Foulkes R, et al. Mechanism of action of certolizumab pegol (CDP870): In vitro comparison with other anti-tumor necrosis factor alpha agents. Inflamm Bowel Dis 2007; 13:1323-32.

5. Ware JE, Snow KK, Kosinski M, Gandek B. SF-36 health survey manual and interpretation guide. Boston, Massachusetts, USA: New England Medical Center/The Health Institute, 1993.

6. Kirwan JR, Reeback JS. Stanford Health Assessment Questionnaire modified to assess disability in British patients with rheumatoid arthritis. Br J Rheumatol 1986; 25:206-9.

7. Ware JE, Kosinski M, Keller SK. SF-36 physical and mental health summary scales: a user's manual. Boston, Massachusetts, USA: New England Medical Center/The Health Institute, 1994.

8. Hewlett S, Hehir M, Kirwan JR. Measuring fatigue in rheumatoid arthritis: a systematic review of scales in use. Arthritis Rheum 2007; 57:429-39.

9. Edward Keystone, Désirée van der Heijde, David Mason, Jr., Robert Landewé, Ronald van Vollenho- ven, Bernard Combe, Paul Emery, Vibeke Strand, Philip Mease, Chintu Desai, and Karel Pavelka. Certolizumab Pegol Plus Methotrexate Is Significantly More Effective Than Placebo Plus Methotrexate in Active Rheumatoid Arthritis Findings of a FiftyTwo-Week, Phase III, Multicenter, Randomized, Double-Blind, Placebo-Controlled, Parallel-Group Study. Arthritis \& Rheumatism November 2008; 58(11):3319-3329.

10. J Smolen, R B Landewé, P Mease, J Brzezicki, D Mason, $\mathrm{K}$ Luijtens, R F van Vollenhoven, A Kavanaugh, M Schiff, G R Burmester, V Strand, J Vencovsky, D van der Heijde. Efficacy and safety of certolizumab pegol plus methotrexate in active rheumatoid arthritis: the RAPID 2 study. A randomised controlled trial. Ann Rheum Dis 2009; 68:797-804.

11. Michael E. Weinblatt, Roy Fleischmann, Tom W. J. Huizinga, Paul Emery, Janet Pope, Elena M. Massarotti, Ronald F. van Vollenhoven, Jürgen Wollenhaupt, Clifton O. Bingham III, Ben Duncan, Niti Goel, Owen R. Davies and Maxime Dougados. Efficacy and safety of certolizumab pegol in a broad population of patients with active rheumatoid arthritis: results from the REALISTIC phase IIIb study. Rheumatology (Oxford. 2012 Dec; 51(12):2204-14.

12. R Fleischmann, J Vencovsky, R F van Vollenhoven, D Borenstein, J Box, G Coteur, N Goel,7 H-P Brezinschek, A Innes, V Strand. Efficacy and safety of certolizumab pegol monotherapy every 4 weeks in patients with rheumatoid arthritis failing previous disease modifying antirheumatic therapy: the FAST4WARD study. Ann Rheum Dis 2009; 68:805-811.

13. Edward C. Keystone, Bernard Combe, Josef Smolen, Vibeke Strand, Niti Goel, Ronald van Vollenhoven, Philip Mease, Robert Landewé, Roy Fleischmann, Kristel Luijtens and Désirée van der Heijde. Sustained efficacy of certolizumab pegol added to methotrexate in the treatment of rheumatoid arthritis: 2-year results from the RAPID 1 trial. Rheumatology (Oxford). 2012 Sep; 51(9):1628-38.

14. Sokka T, Pincus T. Most patients receiving routine care for rheumatoid arthritis in 2001 did not meet inclusion criteria for most recent clinical trials or American College of Rheumatology criteria for remission. J Rheumatol 2003; 30:1138-46.

15. Zink A, Strangfeld A, Schneider M, et al. Effectiveness of tumor necrosis factor inhibitors in rheumatoid arthritis in an observational cohort study: comparison of patients according to their eligibility for major randomized clinical trials. Arthritis Rheum 2006; 54:3399-407. 